\title{
Use of the resuscitation room for trauma
}

\author{
G. A. O'BYRNE \& G. G. BODIWALA
}

Accident and Emergency Department, Leicester Royal Infirmary, Leicester, England

\section{SUMMARY}

This study was designed to assess the use of the resuscitation room for trauma cases, the information being used to identify particular problem areas and put forward possible solutions. One hundred and fifty consecutive resuscitation room trauma patients were studied prospectively, with details being obtained both at the time of admission and $48 \mathrm{~h}$ later.

The results have highlighted the importance of all patients with major trauma being assessed and treated by senior medical staff. In addition, the presence of more than one doctor when managing any resuscitation room trauma patient was shown to be beneficial. Many cases were seen by SHO only, and there is a need for more senior medical staff to be informed of every trauma patient entering the resuscitation room. Recording of vital signs was found to be less than satisfactory and there is a necessity for a departmental policy on this.

\section{INTRODUCTION}

A prospective study of 150 consecutive trauma cases brought into the resuscitation room of the Accident and Emergency (A \& E) Department at the Leicester Royal Infirmary, Leicester, England, was undertaken. The aims were to ascertain the nature and severity of the trauma cases dealt with in a large city in mainland Britain, and to assess the efficacy of the management in the light of the staffing allocation and departmental policy.

\section{METHOD}

One hundred and fifty consecutive patients with injuries thought to necessitate

Correspondence: Mr G. G. Bodiwala, Consultant in Charge, Accident and Emergency Department, Leicester Royal Infirmary, Leicester LE1 SWW, England. 
treatment in the resuscitation room of the A \& E Department at the Leicester Roya Infirmary were studied in a 4-month period from April to August 1985.

Information was gathered on pre-hospital details. From the ambulance information sheet the time of call, time of arrival of the ambulance at the scene, time of departure from the scene and time of arrival at the hospital were obtained. It was also noted $\overrightarrow{\vec{F}_{\vec{f}}}$ whether the Hospital Accident Flying Squad was involved or not, and what treatment? was given on site. All patients' records were logged in a book specifically kept for this․ㅡㄴ purpose. Information on these patients was collected as follows: age; sex; date and time of arrival; nature of incident; status of nursing and medical staff involved, with time seen; vital signs; level of consciousness; investigations; provisional diagnoses; drugs and ${ }^{\text {s }}$ fluids administered; and size of cannulae used. The outcome of management was noted $\vec{P}$ down by the A \& E staff involved, along with the final disposal of the patient and the $\vec{\omega}$ time the patient left the resuscitation room. Information was also obtained on other specialists involved, with the time they were called and the time they arrived in the department.

A final review of injuries sustained was carried out $48 \mathrm{~h}$ after arrival, using the in patient notes. Abbreviated Injury Scale (AIS) score (Greenspan et al., 1985) and Injuryo Severity Score (ISS) were assessed and recorded.

\section{RESULTS}

The average resuscitation room trauma admission rate was approximately nine patie per week. The total number of trauma cases presenting to the department during thing above period was 22600 . Thus $0.66 \%$ of all trauma cases were managed in the resuscitation room.

Eighty-four per cent of the patients in the study were male and $16 \%$ female. There was a marked preponderance of patients in the 16-20 year age group.

The time between the emergency call being made and the patient arriving at the hospital was within $30 \mathrm{~min}$ in $44 \%$ of cases and within $40 \mathrm{~min}$ in $67 \%$ of cases. Thirteen patients took more than $1 \mathrm{~h}$ and, of these six were trapped in vehicles, two were travelling from a long distance, one had severe pain resulting in a very slow journey, and the remaining four patients spent a long time at the scene of the accident but the reasonș. were not known.

Eleven patients arrived under the care of the Hospital Accident Flying Squad, and most of these had been trapped in their vehicles for a while, commonly by the legs. The average ISS of these 11 patients was 29.

Fifty-five per cent of arrivals were during $08.30-17.30,27 \%$ between $17.30-24.00$ 元 and $18 \%$ during midnight -08.30 . Peak times were at $10 \mathrm{am}, 3 \mathrm{pm}, 6 \mathrm{pm}$ and $12 \mathrm{pm}$.

Road traffic accidents accounted for nearly $60 \%$ of all cases, and accidents in the home for $22 \%$

\section{Clinical presentation}

Records of the 150 patients studied revealed that six had no pulse or blood pressure 


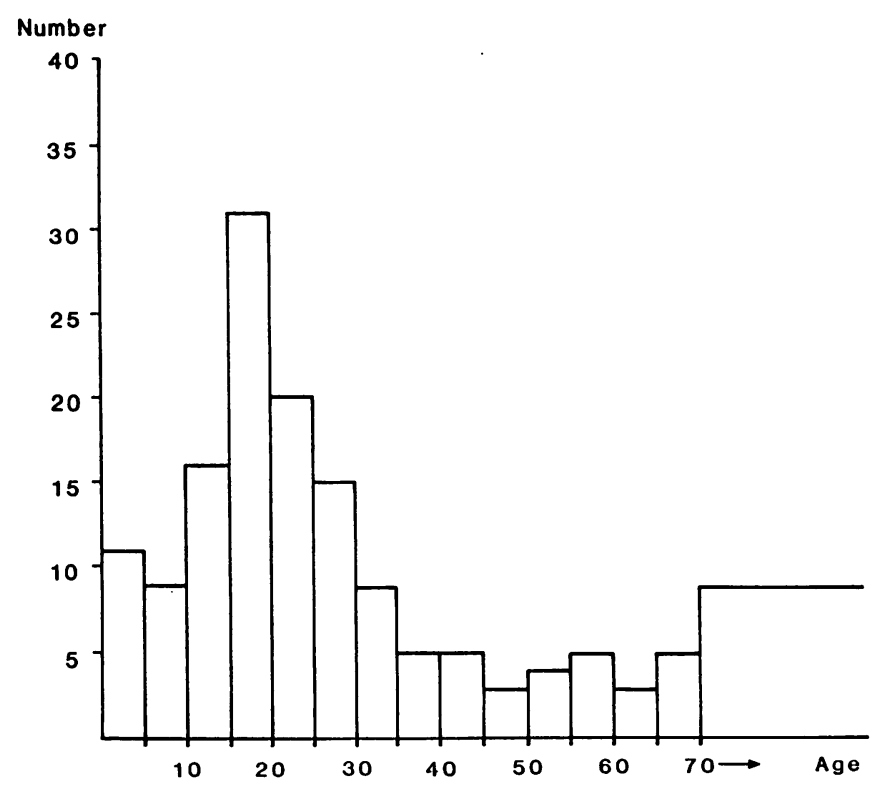

Fig. 1 Numbers and ages of patients.

recorded on arrival, a further two had no pulse recorded and another six had no blood pressure recorded on arrival. Thus, only 136 patients had both pulse and blood pressure recorded on arrival and, moreover, 36 of these had no vital signs recorded subsequently. For the 100 cases who had regular recording of pulse and blood pressure, the interval was greater than $15 \mathrm{~min}$ in 41 cases.

Seventy-one of the total cases had a sister or charge nurse in charge and 75 were managed by a staff nurse. Four cases had an enrolled nurse as head of the nursing team and, in all four cases, the pulse and blood pressure were recorded on arrival, but two of the four had no subsequent regular recordings.

Table 1 Nature of incident and number of cases

\begin{tabular}{lcr}
\hline Nature of incident & $\begin{array}{c}\text { No. of } \\
\text { cases }\end{array}$ & $(\%)$ \\
\hline Accident: RTA & 89 & $(59 \cdot 3)$ \\
$\quad$ Home & 33 & $(22 \cdot 0)$ \\
$\quad$ Sport & 9 & $(6 \cdot 0)$ \\
$\quad$ Work & 9 & $(6 \cdot 0)$ \\
Assault & 4 & $(2 \cdot 7)$ \\
Self-inflicted & 2 & $(1 \cdot 3)$ \\
Unknown & 4 & $(2 \cdot 7)$ \\
\hline
\end{tabular}


Looking at the arrivals between $08.30-17.30$, namely 83 cases $(55 \%$ of all patients studied), 30 of these were seen by only one doctor, 26 being seen by SHO only and fouto by registrar alone. Nine of these 30 cases had an ISS of 15 and over.

Sixty-four $(43 \%)$ of the 150 patients were managed by SHO only. Where the patientso were seen by more than one $\mathrm{A} \& \mathrm{E}$ doctor, the senior doctor was a registrar in 42 cases and a senior registrar in 33 cases. Further analysis showed that $28 \%$ of patients weref seen by SHO and registrar; $8 \%$ by SHO and Senior Registrar; $13 \%$ by SHO, registraro and senior registrar; $0.7 \%$ by registrar and senior registrar; and $7.3 \%$ by registrar alone. $\frac{\overline{\bar{s}}}{\bar{\sigma}}$

Fifty-one per cent of patients were seen immediately, $64 \%$ were seen within 2 min of arrival, and $75 \%$ within 5 min. Five (3\%) patients waited for more than 30 minutes tos be seen by a doctor: three of these five were initially admitted to cubicles outside theo resuscitation room but were subsequently transferred to the resuscitation room because their general condition suddenly worsened, and for two of the five patients the reasonso for the delay are unknown.

Twenty-three patients were unconscious on admission. Twenty-two of these pre-i sented as head injuries, of which 15 were related to road traffic accidents (RTAs) and eight were related to a fall (one had, in fact, had a CVA). Alcohol levels were not measured. One patient presented with profound hypovolaemic shock following an? RTA. Twelve of the 150 patients were 'inappropriately' allocated to the resuscitation room on retrospective analysis. Half of these cases were uncomplicated (and conscious) head injuries, the other half being a wide variety of non-serious injuries.

\section{Investigations}

The investigations most commonly performed were $\mathrm{X}$-rays and blood tests, the lat being FBC, U \& E, and group and save/cross-match. Other tests included blood glucose⿳亠丷厂巾 and arterial blood gases.

\section{Diagnoses}

When patients were seen by SHO only, in $13(20 \%)$ of the 64 cases, the initial diagnosis differed significantly from the final diagnosis (on the basis of anticipation or recognition of respiratory insufficiency, anticipation and treatment of shock, prevention or minimisation of future disabilities, and anticipation or alleviation of pain). The ISS of thesein 13 cases ranged from 5 to 59, the average being 24. Eight of these 13 cases had an ISS of 15 and over. All 'SHO only' cases with ISS greater than 30 were inadequately managede (on the above basis).

When seen by SHO and Registrar, in four ( $9.5 \%)$ of the 42 cases the initial diagnosis differed significantly from the final diagnosis. The ISS of these four cases ranged from 40 to 40 , the average being 19 .

When seen by Registrar only, in three $(27 \%)$ of the 11 cases, the initial diagnosis differed significantly from the final diagnosis. The ISS of these three cases ranged fromg 9 to 34, the average being 22 .

When seen by senior registrar, in conjunction with more junior staff, in four $(12 \%)$ of the 33 cases, the initial diagnosis differed significantly from the final diagnosis. The ISS of these four cases ranged from 14 to 57 , the average being 34 . 
The commonest cause for under or over diagnosis, whether clinically significant or not, was fractured bones (nearly half of the cases). Less common causes were injuries to ligaments/joints, viscera, and nerve tissue. In only a few cases were injuries to skin and soft tissue, and blood vessels misdiagnosed.

\section{Injury severity score (ISS)}

This was collated using the scoring chart of Greenspan et al. (1985). The range of ISS was 1-59, and the average was 16. Fifty-nine (39\%) cases had an ISS of 15 and over, that is, had major trauma (Lowe et al., 1986), and 30 of these presented during 08.3017.30. Eight (5\%) cases had an ISS of greater than 40 and four of these died (before review at $48 \mathrm{~h}$ ). Twenty-five $(39 \%)$ of the 64 cases seen by 'SHO only' had major trauma. In particular, eight $(31 \%)$ of the 26 patients seen by 'SHO only' during the period 08.30-17.30 had major trauma.

Analysis of the body regions involved showed that the 'Head and Neck' group was most commonly affected but had only a moderate average AIS (abbreviated injury scale) value, and the 'Abdomen' group was least commonly affected but had the highest average AIS value. Taking all body regions together, the 11-15 age group had the highest average AIS value and the 56-60 age group had the lowest average AIS value.

\section{Treatment}

One hundred and thirty-four of the 150 patients had one or more intravenous cannulae inserted. Thirty-five of these 134 were children, and 99 were adults. Looking at the adult cases: in 38 , size $18 \mathrm{G}$ cannula was used; in 55, size $16 \mathrm{G}$ cannula; and, in only two cases, size $14 \mathrm{G}$.

No fluid replacement was necessary in 64 of the 150 cases. In those 86 patients where fluids were given (by the intravenous route), some patients received more than one type of fluid. Haemaccel was used in 60 cases, N. Saline in 37 cases and PPF in four cases. Blood was only necessary in the resuscitation room in nine cases and, of these, only one required uncrossmatched blood. Fifty of the 59 patients with major trauma required intravenous fluids.

Specialist teams were called for 130 of the patients, with more than one team being called for some patients. They were called within $30 \mathrm{~min}$ in $53 \%$ of instances, between $30-60$ minutes in $25 \%$ of instances and after more than $60 \mathrm{~min}$ in $22 \%$ of instances.

In 30 cases, the specialist team arrived within $2 \mathrm{~min}$, in 45 cases they took between 2$5 \mathrm{~min}$, and in 32 cases they took between $6-10 \mathrm{~min}$. Thus, in $68 \%$ of cases, the specialist team arrived within $10 \mathrm{~min}$ of being called.

Each patient had the time span recorded between entering and leaving the resuscitation room. Twenty-eight per cent of all patients had left by $1 \mathrm{~h}$, and $55 \%$ of all patients had left by $2 \mathrm{~h}$. Total time spent in the resuscitation room was significantly reduced when more than one $\mathrm{A} \& \mathrm{E}$ doctor saw the patient.

\section{Disposal}

Twelve per cent of patients were transferred directly to operating theatres, $6.5 \%$ 
straight to ITU, $6.5 \%$ were transferred to the burns unit, $8 \%$ to the A \& $\mathrm{E}$ ward, $2 \% \operatorname{to}$ the fracture clinic and $54 \%$ to the general wards. Of the remaining $11 \%$ of patients, five cases needed transfer to the Regional Neurosurgical Centre in another hospital direct from our resuscitation room because of the severity of the injury, two patients died in? the resuscitation room (one from profuse uncontrollable haemorrhage, the other from $\overrightarrow{\mathrm{F}_{5}}$ combination of marked blood loss and severe head injury) and nine patients were welf $\vec{b}$ enough to be discharged home. Of these latter nine patients, five required resuscitation room assessment because of their mode of presentation (for example, marked chest pain $\bar{s}$ of no subsequent significance) but were subsequently found to have no serious problems, and the other four had minor injuries and had been inappropriately allocated to the resuscitation room. The average ISS of these nine patients was $3 \cdot 5$.

The overall in-patient mortality was $8.66 \%$ (13 cases), of which $1.33 \%$ (two cases) $\overrightarrow{\vec{c}}$

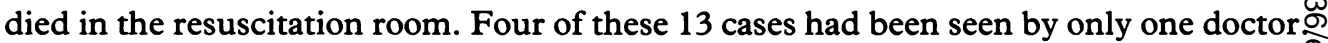
A senior doctor was in attendance in four of the remaining nine cases.

\section{DISCUSSION}

The department sees approximately 110000 patients per year and serves an area of roughly $32-\mathrm{km}$ radius. This area also includes two motor racing circuits, a gravel-pit. based underwater diving school, motorways and major trunk roads. The m迹两 industry in the area is the textile industry.

The department has two consultants, two senior registrars, three registrars and casualty officers who are all senior house officers. The SHOs are allocated to the resuscitation room on a regular basis during their 6-month post and so receive experience in dealing with major trauma. They also participate in the accident flying squad administered by the department and, in addition, they have lectures on how to deal with severely injured and seriously ill patients. The registrars all have a post? graduate qualification, and continuous registrar cover is available in the departmento $24 \mathrm{~h}$ a day. A registrar is present in the department from 08.00 onwards until such timeafter midnight that the department has quietened down, and then based in an on-calB.B. room in the hospital up to 08.00 the next day. The senior registrars are in the department from 08.30-17.30, being on call from home the remainder of the time.

The departmental staffing policy appears to correlate reasonably with the facts thaf $55 \%$ of all resuscitation room trauma cases were admitted between 08.30-17.30 and $51 \%$ of the major trauma cases presented during these hours.

Twenty per cent of the patients seen by 'SHO only' had clinically significant differences between the initial and final diagnoses, and over half of these had majof trauma. In particular, all 'SHO only' cases with ISS greater than 30 were inadequately managed. Surprisingly, the registrars performed no better, with $27 \%$ of their patient延 having clinically significant differences between the initial and final diagnoses However, only $12 \%$ of the patients seen by senior registrar, in conjunction with juniox? staff, had clinically significant differences between the initial and final diagnoses despite the fact that the average ISS of these patients was markedly higher than of those 
patients seen by SHO only or registrar alone. Thus, it is very important that a senior doctor is involved in the management of such cases, especially those with major trauma.

For those situations where a senior $\mathrm{A} \& \mathrm{E}$ doctor is not readily available, it is worth noting that, when a patient was seen by both an SHO and registrar, the percentage of cases where the difference in the initial and final diagnoses was clinically significant decreased by over $50 \%$. In addition, the length of time the patient spent in the resuscitation room was significantly reduced by the presence of more than one doctor.

It is evident from the study that a significant number of patients were seen by SHO alone without involving another doctor, despite the ready availability of a registrar at all times and a senior registrar during 08.30-17.30. In fact, the reason for this was a lack of communication between nursing staff and SHOs, and the more senior medical staff, in that the latter were not informed of the presence of a trauma patient in the resuscitation room.

It is pertinent that it is here in the $\mathrm{A} \& \mathrm{E}$ departments that resuscitation of all trauma patients is carried out and so appropriate training of the junior staff must be considered, as highlighted by Dearden \& Rutherford (1985).

Another prominent result of the survey was how resuscitation room trauma patients did not always have their vital signs recorded (assuming that they were done) and those that did have them recorded had them done too infrequently. No one particular nurse is responsible for taking the vital signs and it is anticipated that this task will be delegated by the nurse in charge.

\section{CONCLUSIONS}

The results have highlighted the importance of all patients with major trauma being assessed and treated by senior medical staff.

The presence of more than one doctor when managing any resuscitation room trauma has been shown to be beneficial in reducing the incidence of clinically significant missed diagnoses and lessening the time the patient spends in the resuscitation room.

In the light of so many cases ( $43 \%$ ) being seen by SHO only, there is a need for more senior medical staff to be informed of every trauma patient entering the resuscitation room, as it is in this way that the senior doctors can assess and treat the major trauma, and more than one doctor will see each resuscitation room trauma patient.

Over half of all resuscitation room trauma presented during daytime hours (08.3017.30), a valuable consideration when planning departmental staffing levels.

Recording of vital signs was found to be less than satisfactory and there is a need for a departmental policy on the recording of these, with guide-lines on what and how frequently to observe, for which a nurse should be identified.

\section{REFERENCES}

Dearden C. H. \& Rutherford W. H. (1985) The resuscitation of the severely injured in the accident and emergency department-a medical audit. Injury 16, 249-52. 
Greenspan L., McLellan B. A. \& Greig H. (1985) Abbreviated injury scale and injury severity score: a scoring chart. Fournal of Trauma 25, 60-4.

Lowe D. K., Oh G. R., Neely K. W. \& Peterson C. G. (1986) Evaluation of injury mechanism as a criterion in trauma triage. The American fournal of Surgery 152, 6-10.

Received 10 fuly 1986; accepted 1 December 1987 